\title{
Langmuir
}

pubs.acs.org/Langmuir

(C) 2010 American Chemical Society

\section{Exact Analytical Expressions for the Potential of Electrical Double Layer Interactions for a Sphere-Plate System}

\author{
Shihong Lin and Mark R. Wiesner* \\ Duke University, Department of Civil and Environmental Engineering, P.O. Box 90287, Durham, North \\ Carolina 27708, United States
}

Received July 30, 2010. Revised Manuscript Received September 24, 2010

\begin{abstract}
Exact, closed-form analytical expressions are presented for evaluating the potential energy of electrical double layer (EDL) interactions between a sphere and an infinite flat plate for three different types of interactions: constant potential, constant charge, and an intermediate case as given by the linear superposition approximation (LSA). By taking advantage of the simpler sphere-plate geometry, simplifying assumptions used in the original Derjaguin approximation (DA) for sphere-sphere interaction are avoided, yielding expressions that are more accurate and applicable over the full range of $\kappa a$. These analytical expressions are significant improvements over the existing equations in the literature that are valid only for large $\kappa a$ because the new equations facilitate the modeling of EDL interactions between nanoscale particles and surfaces over a wide range of ionic strength.
\end{abstract}

\section{Introduction}

Evaluating the potential for electrical double layer (EDL) interactions is of crucial importance in predicting the colloidal stability of particles using Derjaguin-Landau-Verwey-Overbeek (DLVO) theory. ${ }^{1}$ An extensive study has been conducted to model the EDL interaction of systems of different geometries as typified by several important ones such as plate-plate, sphere-sphere, and sphereplate systems. Whereas using a numerical method to solve the complete Poisson-Boltzmann (PB) equation describing the potential distribution of the system is always a viable option at arriving at an exact solution for the potential energy of interaction, it is nevertheless not a very practical tool that can be readily used by all interested researchers. Closed-form analytical expressions have thus been sought after, especially for two kinds of geometry: the plate-plate interaction, which is the simplest case and often the theoretical starting point for studying more complicated systems, and the spheresphere interaction, which is the geometry relevant to the study of particle mutual coagulation. The EDL interaction between a spherical particle and a planar surface (conceptualized by an infinitely flat plate) is also important because it is relevant to particle deposition (either onto an immobile surface or onto a much larger flowing collector as in heteroaggregation) ${ }^{2}$ and it also facilitates the interpretation of the results in measuring single particle-planar surface interaction using atomic force microscopy. ${ }^{3}$ Despite its equal importance, the theoretical study of the sphere-plate system was relatively limited mainly because of the fact that the sphere-plate geometry is, to a large extent, a special case of the sphere-sphere geometry with one of the spheres becoming infinitely large. Therefore, the results for the sphere-sphere system might be easily modified to apply to the sphere-plate system.

*Corresponding author. E-mail: wiesner@ duke.edu. Phone: 919-660-5292. Fax: 919-660-5219.

(1) Vervey, E.; Overbeek, J. Theory of the Stability of Lyophobic Colloids; Elsevier: Amsterdam, 1948.

(2) Elimelech, M.; Williams, R.; Gregory, J.; Jia, X. Particle Deposition and Aggregation: Measurement, Modelling and Simulation; Butterworth-Heinemann: Oxford, England, 1998.

(3) Carnie, S.; Chan, D.; Gunning, J. Langmuir 1994, 10, 2993-3009.

(4) Hogg, R.; Healy, T.; Fuerstenau, D. Trans. Faraday Soc. 1966, 62, 1638-1651.

(5) Derjaguin, B. Kolloid-Z. 1934, 69, 155-164.
In the case of constant potential interaction, Hogg et al. ${ }^{4}$ derived an expression for the EDL interaction potential between two dissimilar flat plates with low surface potentials $\left(\psi_{\mathrm{P}}<k_{\mathrm{B}} T\right)$ :

$$
V_{\mathrm{PP}}^{\psi}=\frac{\varepsilon \kappa}{2}\left[\left(\psi_{\mathrm{P} 1}^{2}+\psi_{\mathrm{P} 2}{ }^{2}\right)(1-\operatorname{coth}(\kappa L))+2 \psi_{\mathrm{P} 1} \psi_{\mathrm{P} 2} \operatorname{csch}(\kappa L)\right]
$$

In the same paper, Hogg et al. also derived an expression for the EDL interaction potential between two dissimilar spheres (in both size and surface potential) using the Derjaguin approximation, ${ }^{5}$ which is composed of the Derjaguin construction and geometric simplifications. The Derjaguin construction is the ringwise dissection of the surface of the hemisphere in which the spherical surface was viewed as an integral of infinite ring-shaped flat plates of infinitesimal width, and the potential energy of the EDL interaction between two spheres was calculated by integrating the EDL interaction potential between pairs of parallel plates. In addition, two simplifying assumptions have to be made in order to obtain an analytical expression: First, it was assumed that the curvature of the spherical particle is small so that in a sphere-sphere system such as that depicted in Figure 1A the following condition holds: $h \ll a_{1}$ and $h \ll a_{2}$, leading to ${ }^{5}$

$$
h d h=\frac{a_{1} a_{2}}{a_{1}+a_{2}} \mathrm{~d} H
$$

Second, when integrating eq 1 along the spherical surface, the upper limit of integration was set to infinity. These simplifying assumptions lead to limited applicability of the expressions so that the expressions are applicable only when $\kappa a \gg 1$ (thin double layer). ${ }^{6}$

With the Derjaguin approximation, the expression for the potential of EDL interaction for a sphere-sphere system was obtained:

$$
\begin{gathered}
V_{\mathrm{SS}}^{\psi}=\frac{\pi a_{1} a_{2}}{a_{1}+a_{2}}\left[\left(\psi_{\mathrm{S} 1}^{2}+\psi_{\mathrm{S} 2}^{2}\right) \ln \left(1-e^{-2 \kappa H_{0}}\right)\right. \\
\left.+2 \psi_{\mathrm{S} 1} \psi_{\mathrm{S} 2} \ln \left(\frac{1+e^{-\kappa H_{0}}}{1-e^{-\kappa H_{0}}}\right)\right]
\end{gathered}
$$




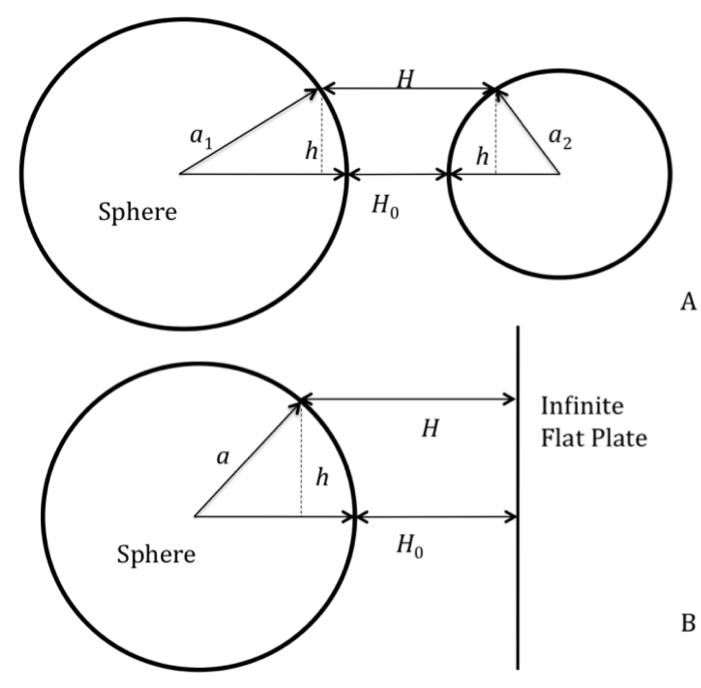

Figure 1. Schematic representation of the geometry for (A) a sphere-sphere interaction and (B) a sphere-plate interaction.

Equation 3 is also called the Hogg, Healy, and Fuerstenau (HHF) expression, and it is widely used in modeling the aggregation of colloidal particles. In addition to the low-potential requirement, significant limitations arise as a consequence of the small-curvature assumption so that the applicability of the HHF expression demands that $\kappa a_{1}, \kappa a_{2} \gg 1 .^{6}$

To calculate the potential of EDL interactions between a sphere and an infinite plate, it is natural to modify the HHF expression for sphere-sphere interaction (HHF-SS) by taking one of the particle sizes to infinity:

$$
\begin{aligned}
V_{\mathrm{SP}}^{\psi}= & \pi \varepsilon a\left[\left(\psi_{\mathrm{sph}}^{2}+\psi_{\mathrm{pl}}^{2}\right) \ln \left(1-e^{-2 \kappa H_{0}}\right)\right. \\
+ & \left.2 \psi_{\mathrm{sph}} \psi_{\mathrm{pl}} \ln \left(\frac{1+e^{-\kappa H_{0}}}{1-e^{-\kappa H_{0}}}\right)\right]
\end{aligned}
$$

Although often employed ${ }^{7-11}$ for its simplicity, such an expression obtained by modifying the expressions for sphere-sphere geometry by taking one of the radii to infinity would inevitably inherit the simplifying assumptions and thus the limited applicability associated with the DA, which would make them less accurate when being applied to the case of a thick double layer (small particle and/or low ionic strengths). Bhattacharjee applied a powerful and versatile method called surface element integration (SEI) that can yield an exact evaluation of the EDL interaction potentials. $^{12}$ The results from SEI are perfectly consistent with those obtained by numerically solving the linearized PoissonBoltzmann equation employing the finite element method. In fact, the Derjaguin construction in essence is no more than a specific mathematical treatment of the SEI. However, a closed-form analytical expression has not been available for the sphereplate EDL interaction based on the SEI method, and the computational burden of performing these calculations remains an impediment.

(7) Vaidyanathan, R.; Tien, C. Chem. Eng. Sci. 1991, 46, 967-983.

(8) Tamai, H.; Suzawa, T. J. Colloid Interface Sci. 1982, 88, 372-377.

(9) Matijevi, E.; Ryde, N. J. Adhes. 1995, 51, 1-14.

(10) Gregory, J.; Wishart, A. Colloids Surf. 1980, 1, 313-334.

(11) Elimelech, M.; Omelia, C. R. Langmuir 1990, 6, 1153-1163.

(12) Bhattacharjee, S.; Elimelech, M. J. Colloid Interface Sci. 1997, 193, 273285.
In the following discussion, we propose a simple method for deriving expressions for the potential of EDL interactions for the sphere-plate system using the Derjaguin construction. Instead of starting from the expression from the sphere-sphere interaction that has already been compromised by the simplifying assumptions, it is possible to take advantage of the relatively simple sphere-plate geometry, starting from the expression for the plate-plate interaction and avoiding the simplifying assumptions employed in DA to arrive at an exact analytical expression for the potential of EDL interaction between a sphere and a plate. Such a method can be applied not only to the constant potential interaction but also to the constant charge interaction (based on the linearized PoissonBoltzmann equation) and intermediate interaction as evaluated by the linear superposition approximation (LSA).

\section{Model Derivation}

The general derivation is presented as the following. For the surface of the front and back hemispheres, the following geometric relations hold:

$$
\begin{gathered}
a-\sqrt{a^{2}-h^{2}}=H-H_{0} ; \quad H_{0} \leq H \leq H_{0}+a \\
a+\sqrt{a^{2}-h^{2}}=H-H_{0} ; H_{0}+a+H \leq H_{0}+2 a
\end{gathered}
$$

The geometric variables in the above equations are explained in Figure 1B. Taking the derivative of both sides of eqs 5 and 6 leads to the same relation:

$$
\begin{aligned}
h d h & =\sqrt{a^{2}-h^{2}} \mathrm{~d} H \\
& =\left(a+H_{0}\right) \mathrm{d} H-H \mathrm{~d} H ; \quad H_{0} \leq H \leq H_{0}+2 a
\end{aligned}
$$

Using the Derjaguin construction, the interaction between the sphere and the plate is calculated as

$$
V_{\mathrm{SP}}=\left.\int 2 \pi V_{\mathrm{PP}} h \mathrm{~d} h\right|_{\text {front }+ \text { back }}
$$

$$
\begin{aligned}
V_{\mathrm{SP}}= & 2 \pi\left[\left(a+H_{0}\right) \int_{H_{0}}^{H_{0}+2 a} V_{\mathrm{PP}}(H) \mathrm{d} H\right. \\
& \left.-\int_{H_{0}}^{H_{0}+2 a} V_{\mathrm{PP}}(H) H \mathrm{~d} H\right]
\end{aligned}
$$

Therefore, as long as the expressions for EDL interaction for a plate-plate system are known and simple enough that the integrals in eq 9 would lead to analytical expressions, exact analytical expressions of the sphere-plate interaction can be obtained. Table 1 is a summary of the expressions for the potential of EDL interaction of a plate-plate system, where $\gamma_{x}$ in eq 12 is defined as

$$
\gamma_{x}=\tanh \left(\frac{z e \psi_{x}}{4 k_{\mathrm{B}} T}\right)
$$

Applying eq 9 to the equations in Table 1 leads to the exact analytical expressions for the potential EDL interaction in a sphere-plate system as summarized in Table 2. Detailed steps for the integration can be found in the Supporting Information.
Equations 7 and 8 lead to 
Table 1. Expressions for the Potential of Plate-Plate EDL Interactions of Different Types ${ }^{a}$

\begin{tabular}{|c|c|c|}
\hline type of interaction & expressions & eq \\
\hline constant potential ${ }^{4}$ & $V_{\mathrm{PP}}^{\psi}=\frac{\varepsilon \kappa}{2}\left[\left(\psi_{\mathrm{P} 1}^{2}+\psi_{\mathrm{P} 2}^{2}\right)(1-\operatorname{coth}(\kappa L))+2 \psi_{\mathrm{P} 1} \psi_{\mathrm{P} 2} \operatorname{csch}(\kappa L)\right]$ & 10 \\
\hline constant charge $^{13}$ & $V_{\mathrm{PP}}^{\sigma}=\frac{\varepsilon \kappa}{2}\left[-\left(\psi_{\mathrm{P} 1}^{2}+\psi_{\mathrm{P} 2}^{2}\right)(1-\operatorname{coth}(\kappa L))+2 \psi_{\mathrm{P} 1} \psi_{\mathrm{P} 2} \operatorname{csch}(\kappa L)\right]$ & 11 \\
\hline intermediate $(\mathrm{LSA})^{14}$ & $V_{\mathrm{PP}}^{\mathrm{LSA}}=32 \kappa\left(\frac{k_{\mathrm{B}} T}{z e}\right)^{2} \gamma_{\mathrm{P} 1} \gamma_{\mathrm{P} 2} \exp (-\kappa L)$ & 12 \\
\hline
\end{tabular}
Supporting Information (SI).

Table 2. Exact Analytical Expressions for the Potential of Sphere-Plate EDL Interaction Evaluated Using Three Different Methods ${ }^{a}$

\begin{tabular}{|c|c|c|}
\hline type & expression & eq \\
\hline constant $\psi$ (LPB-based) & $\begin{array}{l}V_{\mathrm{SP}^{*}}^{\psi}=\left[V_{\mathrm{SP}}^{\psi}\left(H_{0}\right)+V_{\mathrm{SP}}^{\psi}\left(H_{0}+2 a\right)\right]+\left\lfloor\beta^{\psi}\left(H_{0}\right)-\beta^{\psi}\left(H_{0}+2 a\right)\right\rfloor \\
\beta^{\psi}(x)=\frac{\pi \varepsilon}{\kappa}\left[\frac{1}{2}\left(\psi_{\mathrm{sph}^{2}}^{2}+\psi_{\mathrm{pl}^{2}}^{2}\right) \operatorname{Li}_{2}\left(e^{-2 \kappa x}\right)+2 \psi_{\mathrm{sph}} \psi_{\mathrm{pl}}\left[\operatorname{Li}_{2}\left(-e^{-\kappa x}\right)-\mathrm{Li}_{2}\left(e^{-\kappa x}\right)\right]\right]\end{array}$ & 13 \\
\hline $\begin{array}{l}\text { LSA } \\
\text { constant } \sigma \text { (LPB-based) }\end{array}$ & $\begin{array}{l}V_{\mathrm{SP}^{*}}^{\mathrm{LSA}}=V_{\mathrm{SP}}^{\mathrm{LSA}}\left(H_{0}\right)+V_{\mathrm{SP}}^{\mathrm{LSA}}\left(H_{0}+2 a\right)+\frac{64 \pi \varepsilon}{\kappa}\left(\frac{k_{\mathrm{B}} T}{z e}\right)^{2} \gamma_{\mathrm{sph}} \gamma_{\mathrm{pl}}\left[-e^{-\kappa H_{0}}+e^{-\kappa\left(H_{0}+2 a\right)}\right] \\
V_{\mathrm{SP}^{*}}^{\sigma}=\left[V_{\mathrm{SP}}^{\sigma}\left(H_{0}\right)+V_{\mathrm{SP}}^{\sigma}\left(H_{0}+2 a\right)\right]+\left[\beta^{\sigma}\left(H_{0}\right)-\beta^{\sigma}\left(H_{0}+2 a\right)\right] \\
\beta^{\sigma}(x)=\frac{\pi \varepsilon}{\kappa}\left[-\frac{1}{2}\left(\psi_{\mathrm{sph}^{2}}^{2}+\psi_{\mathrm{pl}}{ }^{2}\right) \mathrm{Li}_{2}\left(e^{-2 \kappa x}\right)+2 \psi_{\mathrm{sph}} \psi_{\mathrm{pl}}\left[\operatorname{Li}_{2}\left(-e^{-\kappa x}\right)-\mathrm{Li}_{2}\left(e^{-\kappa x}\right)\right]\right]\end{array}$ & $\begin{array}{l}15 \\
16 \\
17\end{array}$ \\
\hline
\end{tabular}

${ }^{a} V_{\mathrm{SP}}^{\psi}, V_{\mathrm{SP}}^{\sigma}$, and $V_{\mathrm{SP}}^{\mathrm{LSA}}$ are the existing expressions for the potential of EDL interaction for a sphere-plate system as modified from those for the sphere-sphere interaction obtained using the Derjaguin approximation. They are also listed in Table S1.

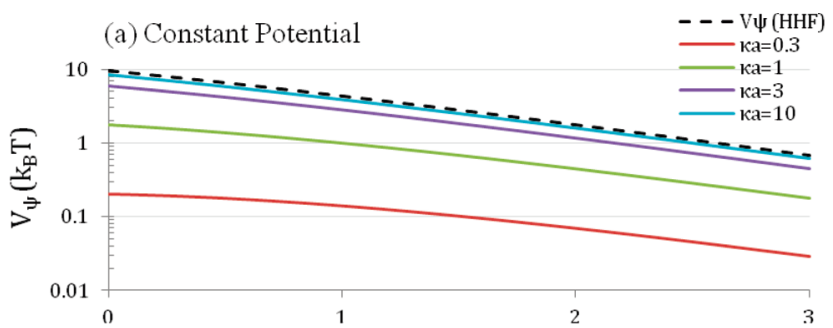

(b) Linear Superposition Approximation
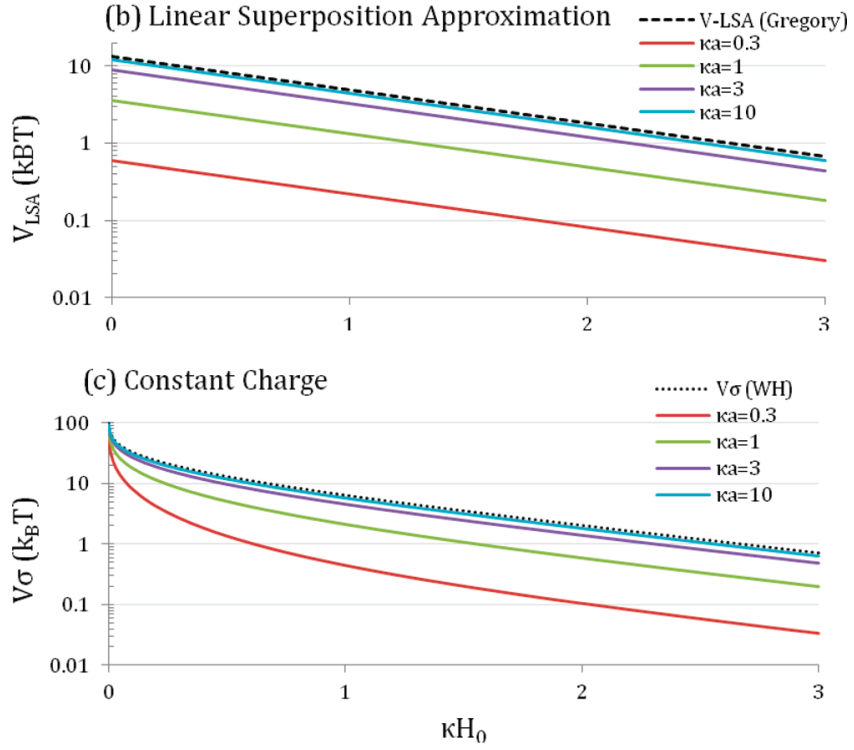

Figure 2. Potential energy for the EDL interaction between a sphere and a plate for three scenarios: (a) constant potential, (b) linear superposition approximation, and (c) constant charge. Colored results are obtained using the corrected expressions presented in Table 2 whereas curves in black are obtained using the uncorrected expressions. The particle size is $10 \mathrm{~nm}$ (radius), and $e \psi_{\mathrm{S}}=e \psi_{\mathrm{P}}=k_{\mathrm{B}} T$.

The function $\operatorname{Li}_{n}(z)$ is the polylogarithm (also known as the de Jonquiere function) of the arguments $n$ and $z$ and is defined as

$$
\operatorname{Li}_{n}(z)=\sum_{k=1}^{\infty} \frac{z^{k}}{k^{n}}
$$

\footnotetext{
(13) Wiese, G.; Healy, T. Trans. Faraday. Soc. 1970, 66, 490-499.
}
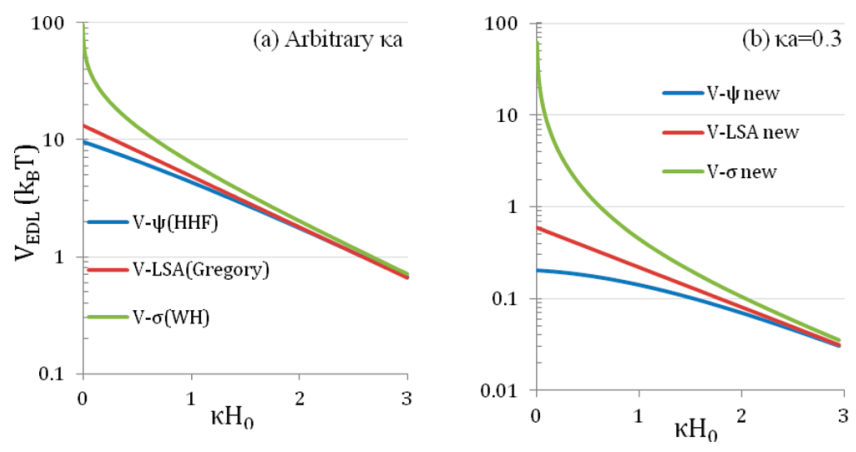

Figure 3. Comparison between potentials of three different types of EDL interactions using both the uncorrected and the corrected expressions. (The expression of the constant charge interaction overestimates, but it is the only available analytical expression for such a sphere-plate interaction.) Parameters for calculation are the same as those described in Figure 2.

When $n=2, \mathrm{Li}_{2}(z)$ is also called the dilogarithm, which has the following integral form: ${ }^{15}$

$$
\mathrm{Li}_{2}(z)=-\int_{0}^{z} \frac{\ln (1-t)}{t} \mathrm{~d} t
$$

All of these expressions in Table 1 can be written in a similar structure as the sum of $V_{\mathrm{SP}}\left(H_{0}\right)$ and $V_{\mathrm{SP}}\left(H_{0}+2 a\right)$ plus a correction term. It should be noted that all of the $V_{\mathrm{SP}}$ terms, regardless of interaction type, are preceded by the particle radius $a$ whereas all of the correction terms are preceded by the Debye length $1 / \kappa$, which clearly explains why $\kappa a$ could serve as the criterion index in determining the applicability of the uncorrected expressions: for a thin double layer $(\kappa a \gg 1)$, the correction terms would become negligible and thus the corrected expressions would be reduced to $V_{\mathrm{SP}}\left(H_{0}\right)+V_{\mathrm{SP}}\left(H_{0}+2 a\right)$. If $a$ is not too small, then $V_{\mathrm{SP}}\left(H_{0}\right)$ would dominate this sum, thereby making the corrected expression close to the uncorrected ones.

It should be kept in mind when applying eq 13 (with eq 14) and eq 16 (with eq 17) that they are based on the linearized PB

(14) Gregory, J. J. Colloid Interface Sci. 1975, 51, 44-51.

(15) Abramowitz, M., Stegun, I., Eds.; Handbook of Mathematical Functions with Formulas, Graphs, and Mathematical Tables; U.S. Government Printing Office: Washington, DC, 1964. 
equation, which demands that the potential everywhere be low. Some researchers argue that a constant potential interaction is unlikely ${ }^{16}$ whereas some point out that a low surface potential cannot be maintained for constant charge interaction. ${ }^{14}$ Now it seems to be agreed on that the intermediate case given by LSA would be the most appropriate approximation for the realistic interaction. ${ }^{14,17}$ However, the appropriateness of each of these equations is beyond the scope of our current discussion.

It is worth pointing out that the results calculated using eq 13 for the constant potential interaction, as presented in Figure 2A, are essentially identical to those given by Bhattacharjee using both SEI and the finite element method, ${ }^{12}$ which further supports the validity of the derived expressions. In addition, it was known that with the uncorrected expressions, the constant potential interaction would always give the lowest potential energy, the constant charge interaction would give the highest, and the potential energy obtained using LSA would fall between that of the other two cases. ${ }^{14}$ It is demonstrated in Figure 3 that such an order for the potential energy in three different cases remains valid for corrected expressions, even when $\kappa a$ is small.

Acknowledgment. This material is based upon work supported by the National Science Foundation (NSF) and the Environmental Protection Agency (EPA) under NSF Cooperative Agreement EF-0830093, Center for the Environmental Implications of NanoTechnology (CEINT). Any opinions, findings, conclusions, or recommendations expressed in this material are those of the author(s) and do not necessarily reflect the views of the NSF or the EPA. This work has not been subjected to EPA review, and no official endorsement should be inferred.

(16) Frens, G.; Engel, D.; Overbeek, J. Trans. Faraday. Soc. 1967, 63, 418-423.

(17) Petosa, A.; Jaisi, D.; Quevedo, I.; Elimelech, M.; Tufenkji, N. Environ. Sci. Technol. 2010, 44, 6532-6549.

\section{Nomenclature}

$\varepsilon=$ dielectric permittivity of the interaction medium

$\kappa=$ Debye constant

$z=$ charge number of the electrolyte

$e=$ electronic charge

$k_{\mathrm{B}}=$ Boltzmann constant

$T=$ temperature

$a_{1}, a_{2}=$ radii of spheres 1 and 2 in a sphere-sphere interaction

$a=$ radius of the sphere in a sphere-plate interaction

$L, H_{0}=$ plate-plate separation and sphere-sphere (sphere-plate) separation

$V_{\mathrm{PP}}, V_{\mathrm{SS}}, V_{\mathrm{SP}}=$ potentials for $\mathrm{EDL}$ interaction for plate-plate, sphere-sphere, and sphere-plate interactions, respectively

$\psi_{\mathrm{sph}}, \psi_{\mathrm{pl}}=$ surface potentials of sphere and plate, respectively, for a sphere-plate interaction

$\psi_{\mathrm{P} 1}, \psi_{\mathrm{P} 2}=$ surface potentials of plates 1 and 2 , respectively, for a plate-plate interaction

Supporting Information Available: Summary of the existing analytical expressions for EDL interaction for a plateplate system, a sphere-sphere system, and a sphere-plate system. Detailed derivation procedures for the proposed exact analytical expressions presented in Table 1. This material is available free of charge via the Internet at http:// pubs.acs.org.

Note Added after ASAP Publication. This article was published ASAP on October 1, 2010. Figure 2, Table 1, and the Supporting Information have been modified. The correct version was published on October 7, 2010. 